\title{
Measurement of electron trapping in the Cornell Electron Storage Ring
}

\author{
M. G. Billing, J. Conway, E. E. Cowan, J. A. Crittenden, ${ }^{*}$ W. Hartung, J. Lanzoni, Y. Li, \\ C. S. Shill, J. P. Sikora, and K. G. Sonnad \\ CLASSE, Cornell University, Ithaca, New York 14850, USA
}

(Received 11 September 2013; published 2 April 2015)

\begin{abstract}
The buildup of low-energy electrons has been shown to affect the performance of a wide variety of particle accelerators. Of particular concern is the persistence of the cloud between beam bunch passages, which can impose limitations on the stability of operation at high beam current. We have obtained measurements of long-lived electron clouds trapped in the field of a quadrupole magnet in a positron storage ring, with lifetimes much longer than the revolution period. Based on modeling, we estimate that about $7 \%$ of the electrons in the cloud generated by a 20 -bunch train of $5.3 \mathrm{GeV}$ positrons with $16-\mathrm{ns}$ spacing and $1.3 \times 10^{11}$ population survive longer than $2.3 \mu \mathrm{s}$ in a quadrupole field of gradient $7.4 \mathrm{~T} / \mathrm{m}$. We have observed a nonmonotonic dependence of the trapping effect on the bunch spacing. The effect of a witness bunch on the measured signal provides direct evidence for the existence of trapped electrons. The witness bunch is also observed to clear the cloud, demonstrating its effectiveness as a mitigation technique.
\end{abstract}

DOI: 10.1103/PhysRevSTAB.18.041001

PACS numbers: 41.75.Ht, 29.20.db, 41.85.Lc, 79.20.Hx

\section{INTRODUCTION}

Electron cloud buildup has been observed in many accelerators since the 1960s [1]. Adverse consequences of electron cloud buildup include emittance growth, beam instabilities, and excess heat load to cryogenic systems.

Positron storage rings for which electron clouds have been an important factor in the design and performance include KEKB in Japan [2] and PEP-II in the USA [3]. Proton accelerators affected by electron clouds include the Los Alamos Proton Storage Ring (PSR) in the USA [4], CERN's Proton Synchrotron (PS), Super Proton Synchrotron (SPS) and Large Hadron Collider (LHC) [5]. At the LHC, electron cloud has been observed to affect the cryogenic heat load [6].

Electron cloud buildup is a major concern for accelerator upgrade programs and for the design of future accelerators. Electron cloud considerations have driven the design of the SuperKEKB collider [7] and the positron damping ring for the proposed International Linear Collider (ILC) [8]. The LHC luminosity upgrade is contingent on reducing the bunch spacing to $25 \mathrm{~ns}$ [9]; at this bunch spacing, severe electron cloud buildup has been observed, such that this bunch pattern has been used for beam scrubbing runs [5]. The success of the upgrade is likely to be contingent on limiting electron cloud buildup.

\footnotetext{
*crittenden@cornell.edu

Present address: KEK, 1-1 Oho, Tsukuba, Ibaraki 305-0801, Japan.

Published by the American Physical Society under the terms of the Creative Commons Attribution 3.0 License. Further distribution of this work must maintain attribution to the author $(s)$ and the published article's title, journal citation, and DOI.
}

Considerable work has been done on the development of electron cloud mitigation techniques. At KEKB and PEP-II, solenoidal magnetic field windings were installed on the beam-pipes. For SuperKEKB, solenoidal windings are used in field-free regions, while TiN coatings and antechambers are included in quadrupole magnets, where solenoidal windings cannot be used. Carbon coatings for the dipole magnet vacuum chambers in the SPS are under study at CERN [10].

The electron cloud is observed to build up during the passage of a train of closely-spaced bunches, imposing restrictions on the operational bunch charge and train length. In field-free regions, gaps between trains allow the electron cloud to dissipate. In regions of magnetic field, however, cloud electrons can become trapped over long periods of time. Since trapped electrons can interact with the beam over many turns, they have the potential for more severe effects.

Electron cloud trapping has been studied experimentally and via simulation. Trapping of electrons oscillating around a 70-m-long proton bunch in the LANL PSR storage ring has been observed. [4]. At LBNL, electrons were observed to be trapped in the fields of an ion beam and accelerator elements, and measurements of the time dependence of electron cloud buildup were carried out [11]. Estimates of long-lived electron cloud buildup at the LHC and consequences for vacuum chamber heat load have been presented in Ref. [12]. More recently, heat load in the final-focus quadrupoles of the LHC has been attributed to electron cloud buildup [13]. Simulations were used to study electron trapping in quadrupole and sextupole magnets for the parameters of the KEKB positron ring [14], as well as for the Cornell Electron Storage Ring (CESR) and the ILC 
positron damping ring [15]. Prior to the measurements presented here, no experimental study of electron trapping in a positron storage ring has been available to validate modeling efforts.

A principal goal of the Cornell Electron Storage Ring Test Accelerator program [16] is to investigate performance limitations in future high-energy low-emittance rings. These studies include measurements of electron cloud buildup caused by synchrotron-radiation-induced photoemission on the surface of the vacuum chamber. The CESR ring stores positron and electron beams of energy $1.8 \mathrm{GeV}$ to $5.3 \mathrm{GeV}$, arranged in bunches spaced in intervals of 4 or $14 \mathrm{~ns}$, with bunch populations ranging up to $1.6 \times 10^{11}$. A variety of detectors sensitive to cloud electrons incident on the vacuum chamber wall have been used to study cloud buildup [17-21].

The potential for undesired consequences to accelerator performance motivated the study of electron trapping in the CESRTA electron cloud research program. In this paper, we report on the measurement of electron trapping in a quadrupole magnet over a $2.3 \mu$ s time interval between bunch train passages. Our demonstration of cloud trapping is based on two observations: first, the revolution-averaged electron flux arriving at the vacuum chamber wall during the passage of a ten-bunch train of positrons is greater when a second such bunch train immediately follows, showing that cloud is present at the time of arrival of the first tenbunch train; second, inserting a single positron bunch over a broad time range centered halfway around the ring reduces the observed flux of electrons at the wall during the train passage, showing that trapped electrons were cleared by the intermediate bunch. It is noteworthy that beam-free intervals in the ring are ineffective at clearing the electrons, since the trapping mechanism is not contingent upon the beam potential as was the case at the PSR.

\section{TIME-RESOLVING ELECTRON DETECTOR}

Time-resolving electron detectors have provided detailed information on local cloud formation, allowing the independent characterization of photoelectron and secondary electron production mechanisms [20,21]. We have installed shielded detectors in a cylindrical stainless steel vacuum chamber of inner diameter $95.5 \mathrm{~mm}$ inside a 60-cm-long quadrupole magnet, as shown in Fig. 1a. One detector was located longitudinally near one end of the iron yoke in order to measure electron cloud buildup in the fringe field. In the following, we refer exclusively to measurements obtained from the detector positioned in the longitudinal center of the magnet and located in azimuth at 45 degrees from the horizontal midplane toward the inside of the ring, as shown in Fig. 1b. Electrons are collected on the 10-mm-wide copper trace (Fig. 1c) which tapers to a transmission line using the grounded copper on the other side of the 0.12-mm-thick Kapton sheet. The total length of the trace including the 10 -mm-wide, 102-mm-long rectangular central region is
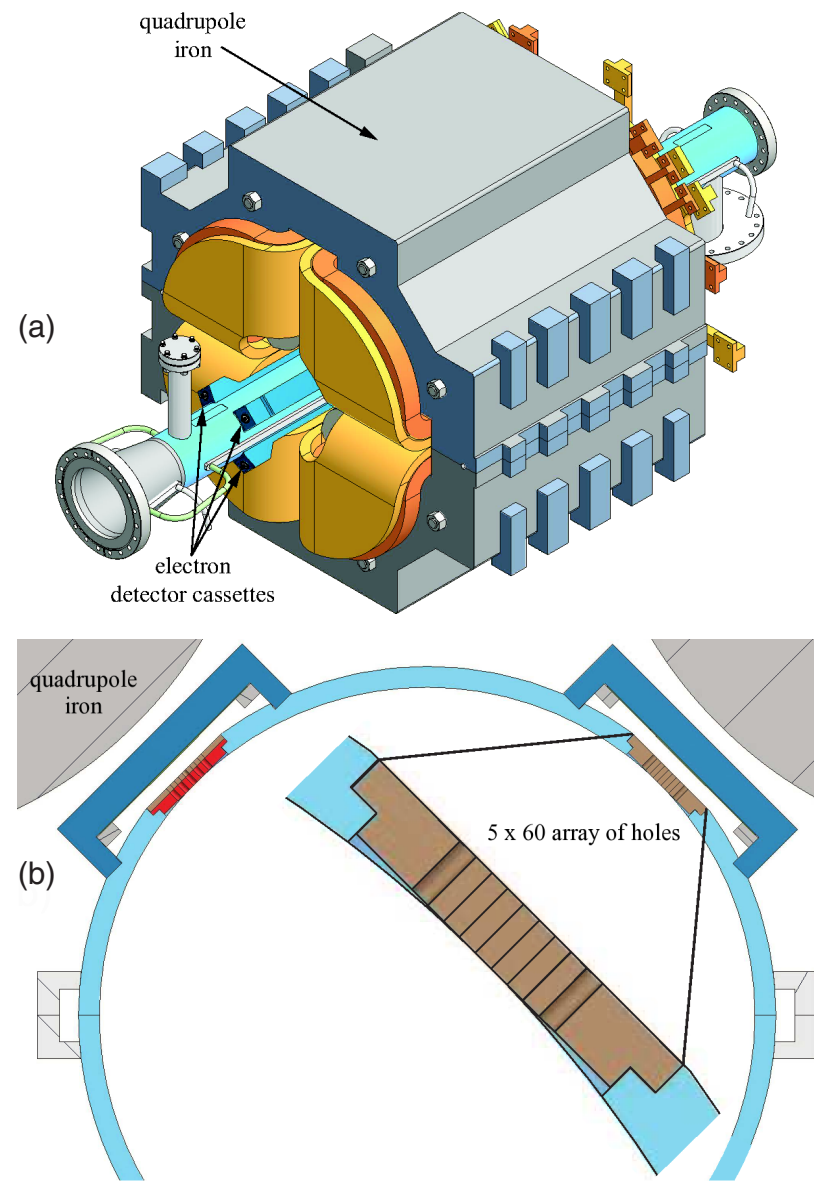

(c)

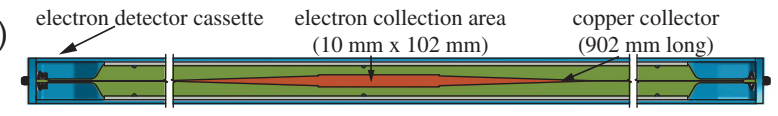

(d)

$5 \times 60$ array of holes $(0.8 \mathrm{~mm}$ diameter $)$

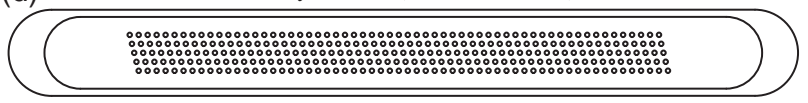

FIG. 1. (a) Vacuum chamber equipped with electron detectors in the quadrupole magnet. (b) Arrangement of two detectors in front of the magnet poles as seen from the positron arrival direction. (c) Geometry of the copper electrode biased at $50 \mathrm{~V}$ to collect electrons entering through the pattern of holes in the beam-pipe shown in (d). The rectangular region of the collector and the pattern of holes are each about $10 \mathrm{~cm}$ long.

$907 \mathrm{~mm}$. The pattern of $5 \times 60$ parallel 0.8 -mm-diameter holes shown in Fig. 1d allows passage of cloud electrons through the beam-pipe to the collector. The chosen hole diameter gives a depth-to-diameter ratio of $3: 1$ in order to shield the detector from the rf power radiated by the $18-\mathrm{mm}-$ long positron bunches [22]. The hole pattern is $7.1 \mathrm{~mm}$ wide and $94.4 \mathrm{~mm}$ long. Figure 2 shows a schematic view of the beam-pipe, hole pattern and detector arrangement.

The collector is biased at $+50 \mathrm{~V}$ relative to the vacuum chamber in order to prevent secondary electrons from leaving the collector surface. The AC-coupled front-end 


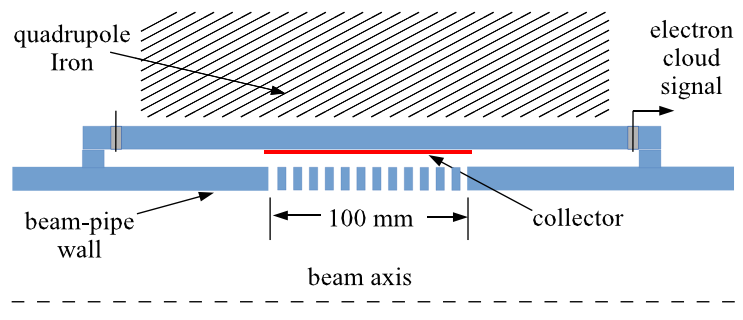

FIG. 2. Schematic cross section of the electron detector, which is located near the longitudinal center of the quadrupole magnet. The holes in the beam-pipe wall allow cloud electrons to reach the collector.

readout electronics consists of two Mini-Circuits ZFL-500 broadband amplifiers with $50 \Omega$ input impedance and a total gain of $40 \mathrm{~dB}$. Oscilloscope traces are digitized to 8-bit accuracy in 1000 time bins, typically 0.5 or $1.0 \mathrm{~ns}$ wide, averaging over 8000 beam-synchronous triggers. The direct beam-induced signal from the residual transmission of high-frequency rf power through the shielding holes results in a damped ringing in the raw oscilloscope signals. All signals depicted in the figures below show the result of applying a 13-MHz low-pass digital post-processing filter which suppresses this noise by an order of magnitude.

Figure 3 shows the filtered signals for 10- and 20-bunch trains of $5.3 \mathrm{GeV}$ positrons. The bunches have rms sizes of $1.8 \mathrm{~mm}$ horizontally and $0.08 \mathrm{~mm}$ vertically. The average bunch population is $1.3 \times 10^{11}$. The bunch spacing is $14 \mathrm{~ns}$ and the bunch-to-bunch population is uniform to a few percent. The quadrupole field gradient is $7.4 \mathrm{~T} / \mathrm{m}$, horizontally focusing.

The larger signal during the first 10 bunches of the 20-bunch train relative to that for the 10-bunch train shows the presence of cloud prior to the arrival of the train. One can deduce that electrons remain trapped at least as long as the $2.3 \mu \mathrm{s}$ beam-free interval prior to the return of the bunch train. The decrease in cloud buildup rate following

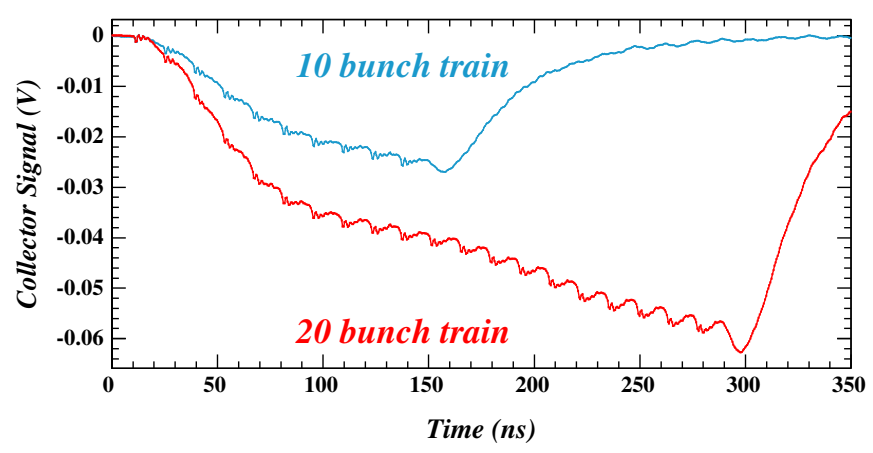

FIG. 3. Electron detector signals recorded for 10- and 20-bunch trains of $5.3 \mathrm{GeV}$ positrons for an average bunch population of $1.3 \times 10^{11}$. The enhanced signal during the first 10 bunches of a 20-bunch train relative to that for the 10-bunch train shows that electrons were trapped during the entire $2.3 \mu$ s interval prior to the return of the bunch train.

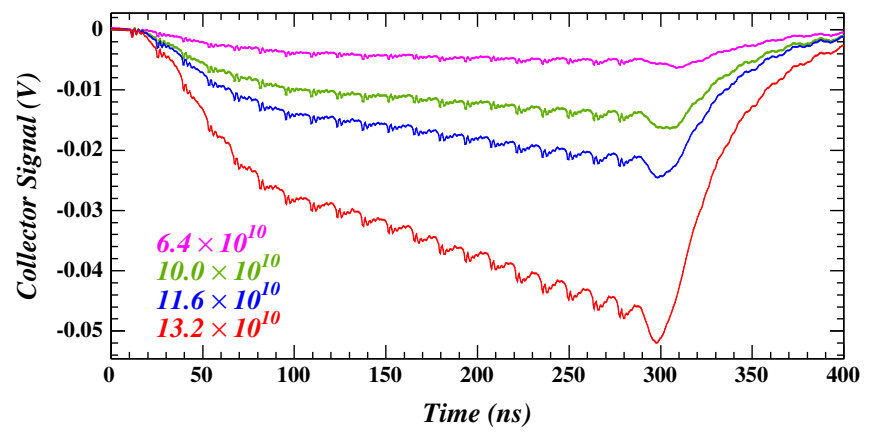

FIG. 4. Dependence of the signals on bunch population for 20-bunch trains with 14 ns spacing. The dependence is strongly nonlinear, the signal amplitude increasing by an order of magnitude for a factor of two increase in bunch population.

the first 6 bunches indicates that a subset of trapped electrons which can contribute signal has become depleted at that time. In spite of this clearing of the trapped reservoir of electrons, the signal does not return to the level of the 10-bunch signal, showing that the additional cloud seeded by the long-term trapping is self-sustaining. The signal depends strongly on the bunch population, decreasing by an order of magnitude as the bunch population decreases by a factor of two from $1.3 \times 10^{11}$ to $6.4 \times 10^{11}$, as shown in Fig. 4 .

The dependence of trapping on the bunch spacing is shown in Fig. 5 for a bunch population of about $1.3 \times 10^{11}$. The decrease with increasing bunch spacing can be understood in terms of an overall decrease in cloud buildup. However, the enhancement of the signal at 16-ns spacing relative to the signal for 14-ns spacing shows that when electron trapping is of concern, care must be taken in the choice of bunch spacing.

We have investigated the effectiveness of an intermediate bunch as a mechanism for clearing the trapped cloud. Figure 6 shows the three signals obtained from (i) a 20-bunch train, (ii) a 20-bunch train with a clearing bunch following about $900 \mathrm{~ns}$ after the end of the train, and (iii) a

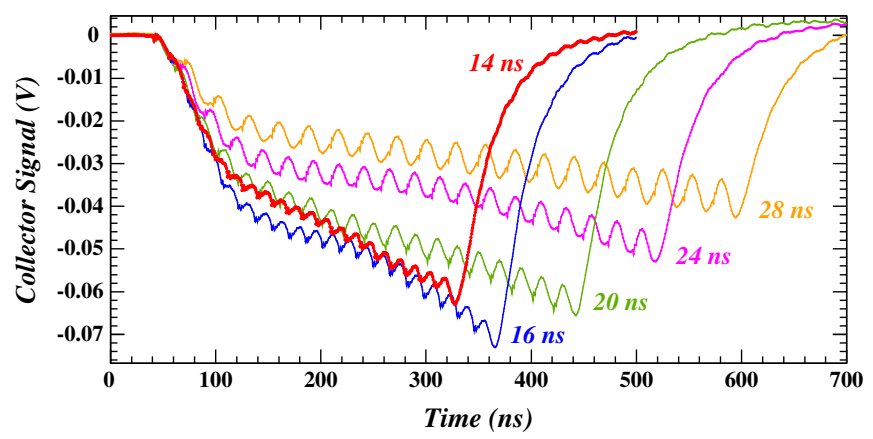

FIG. 5. Comparison of signals obtained from 20-bunch trains with spacing 14, 16, 20, 24 and $28 \mathrm{~ns}$. The increase in signal for the 16-ns spacing relative to the 14-ns spacing shows that longterm cloud electron trapping can be enhanced by an unfortunate choice of bunch spacing. 


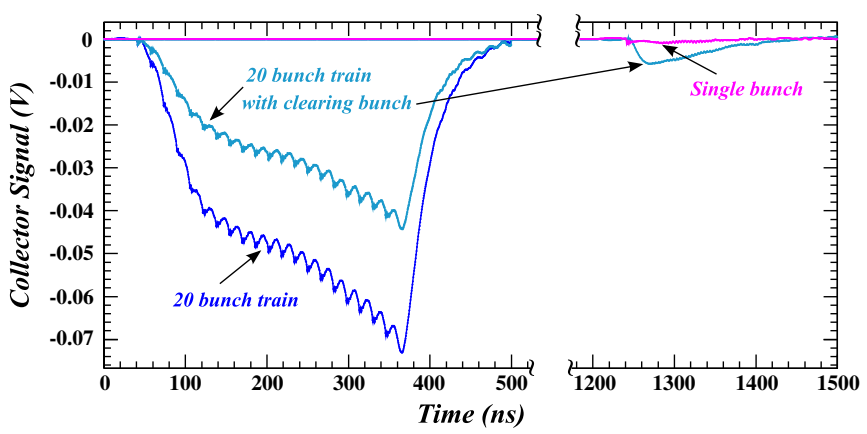

FIG. 6. Effect of an intermediate clearing bunch following about $900 \mathrm{~ns}$ after the end of a 20-bunch train for the case of 16-ns spacing. The difference in magnitude between the signals at $1250 \mathrm{~ns}$ is directly sensitive to the trapped electrons produced by the 20-bunch train.

single bunch. The single-bunch signal is plotted to coincide with the signal from the clearing bunch for the purpose of comparison. The clearing bunch accelerates trapped cloud electrons into the detector, and thus provides direct evidence for the trapped cloud. In addition, the reduced signal from the 20-bunch train when the clearing bunch is present shows the effectiveness of such a mitigation technique. We verified that the clearing effectiveness is independent of the delay of the clearing bunch over a range of $\pm 500 \mathrm{~ns}$. The full clearing effect was achieved when the clearing bunch population reached about $20 \%$ of the average population of the bunches in the train.

\section{TRAPPING MECHANISM}

The long-term trapping of electrons in nonuniform fields such as quadrupole fields can be understood in terms of an adiabatic magnetic moment $\mu$ given by

$$
\mu=\frac{m v_{\perp}^{2}}{2 B},
$$

where $m$ is the mass of the electron, $B$ is the magnetic field magnitude, and $v_{\perp}$ is the velocity component perpendicular to the magnetic field vector (see, for example, Ref. [23]). This quantity remains invariant as long as $\frac{d B}{B} \ll 1$ during the cyclotron motion, or, equivalently,

$$
\Gamma=\frac{|\nabla B| r_{\mathrm{c}}}{B} \ll 1,
$$

where $r_{\mathrm{c}}$ is the cyclotron radius. Combining the conditions of conservation of magnetic moment and conservation of energy, one can specify a "velocity-space loss cone" angle, $\Theta_{\mathrm{LC}}$, which defines the trapping condition. A particle moving from a region of lower field to a region of higher field reverses its path if the velocity components perpendicular and parallel to the magnetic field at the starting position, denoted by $v_{\perp}^{\text {in }}$ and $v_{\|}^{\text {in }}$ respectively, are related such that

$$
\frac{v_{\|}^{\text {in }}}{v_{\perp}^{\text {in }}} \leq\left(\frac{B_{\text {bd }}}{B_{\text {in }}}-1\right)^{1 / 2}
$$

Here $B_{\text {in }}$ is the magnetic field magnitude at the start point, and $B_{\mathrm{bd}}$ is the magnitude along the field line at the boundary beyond which the particle is lost. If the above relationship is satisfied, the particle reaches a point where the parallel velocity goes to zero, and the particle reverses its path along the field line. In a quadrupole magnetic field, the trapped particle is confined between two such mirror points located along a field line symmetric about either the horizontal or the vertical axis. While the particle mirrors between the pair of points, it drifts in the longitudinal direction until it reaches the fringe region of the quadrupole, where it can escape [24]. This drift is caused by a nonzero gradient and curvature in the magnetic field, often referred to as the "grad B" and "curvature" drift, respectively. For a $7.4 \mathrm{~T} / \mathrm{m}$ field gradient, the longitudinal drift over the duration of one CESR beam revolution is significant only when the electron energy is of the order of $1 \mathrm{keV}$. The energy distribution obtained from the cloud build-up modeling described below indicate that less than $3 \%$ of the electrons have energies exceeding $1 \mathrm{keV}$.

The cosine of the loss cone angle represents the fractional solid angle in velocity space within which a particle remains confined. Thus, for a localized distribution of isotropic velocities, it represents the probability of confinement at that point. It can be expressed as

$$
P_{\mathrm{tr}}=\cos \Theta_{\mathrm{LC}}=\left(1-\frac{B_{\mathrm{in}}}{B_{\mathrm{bd}}}\right)^{1 / 2}
$$

and is shown in Fig. 7 as a function of horizontal position $x$ along the midplane of the vacuum chamber. The probability of confinement decreases with $x$, the distance from the beam, provided $\Gamma \ll 1$. The adiabatic condition can be expressed as

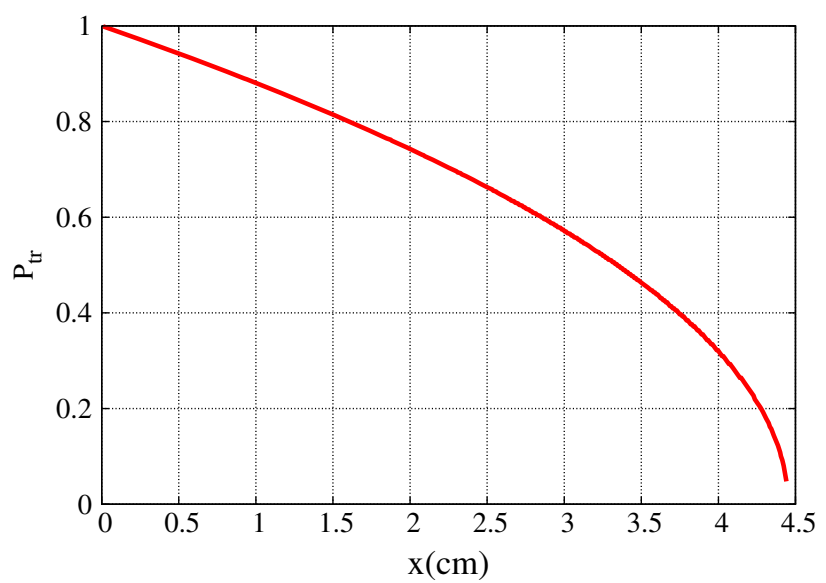

FIG. 7. Cosine of the loss-cone angle, $P_{\text {tr }}$, versus horizontal position in the midplane of the vacuum chamber. The trapping probability increases toward the center of the chamber as long as the adiabaticity condition is satisfied. 


$$
\Gamma=\frac{\sqrt{2 m E_{\perp} / e}}{K x^{2}} \ll 1
$$

where $e$ is the electron charge, $K$ is the quadrupole field gradient and $E_{\perp}$ the kinetic energy corresponding to the velocity component perpendicular to the magnetic field. For electrons in a quadrupole with field gradient $K=7.4 \mathrm{~T} / \mathrm{m}, \Gamma$ reduces to

$$
\Gamma=4.6 \times 10^{-3} \frac{\sqrt{E_{\perp} / \mathrm{eV}}}{(x / \mathrm{cm})^{2}} .
$$

For comparison, the beam kick produced by a bunch carrying $1.3 \times 10^{11}$ positrons on an electron at the vacuum chamber wall is $60 \mathrm{eV}$ in the impulse approximation [25], easily satisfying the trapping condition. On the other hand, an electron with a horizontal momentum of $40 \mathrm{keV} / \mathrm{c}$ located $1 \mathrm{~cm}$ from the beam in the horizontal midplane is likely to hit the chamber wall.

\section{NUMERICAL MODELING OF ELECTRON CLOUD BUILDUP}

We have employed a particle-in-cell, time-sliced cloud buildup modeling code [26] to improve our understanding of the electron trapping mechanism and the observed signals. The code includes simulation algorithms for photoelectron generation, macroparticle tracking in the 2D electrostatic fields of the beam and the cloud, and $3 \mathrm{D}$ tracking in a variety of ambient magnetic fields, as well as for a detailed model of the interaction of cloud electrons with the vacuum chamber surface [27].

The code has been supplemented with response functions for the time-resolving electron detectors [28]. As a function of incident angle and energy, a fraction of the macroparticle charge hitting the wall in the region of the detector contributes to the modeled signal. The fraction is derived from an analytic calculation of the hole acceptance for the case of a magnetic field parallel to the hole axis. For an arbitrary magnetic field strength, the acceptance of the holes is derived by relating the incident kinetic energy and angle to the cyclotron radius and the wall traversal time, i.e., the fractional number of cyclotron revolutions performed in the wall. Thus the acceptance at high field extends to grazing angles of incidence when the cyclotron radius is smaller than the hole radius.

The amplitude of the modeled signal was found to be very sensitive to the assumed secondary emission yield, increasing by an order of magnitude as the peak secondary yield was increased from 1.4 to 1.9. The measured signal amplitude was reproduced with values for the peak secondary yield and elastic yield of 1.4 and 0.5 , respectively.

The model shows the signal to be generated predominantly by electrons originally produced on the field lines entering the detector, i.e., from a narrow surface region in

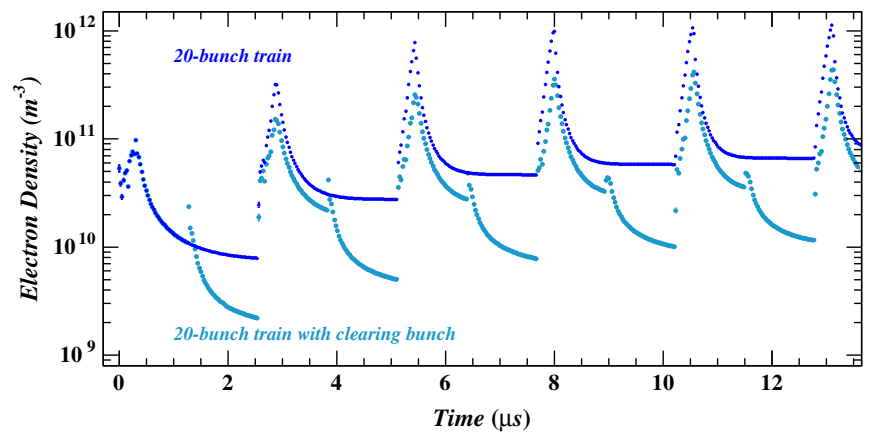

FIG. 8. Results for the beam-pipe averaged cloud density from the numerical model of electron buildup for the case of the $5.3 \mathrm{GeV} 20$-bunch train of positrons with 16-ns spacing shown in Fig. 6, with and without an intermediate clearing bunch.

front of the diametrically opposed pole and from 4-mm-wide regions on the vacuum chamber surface in front of the other two poles extending from the middle of the pole toward the detector. These signal macroparticles spiral around field lines which pass within a few millimeters of the beam. The electrons which remain trapped during the $2.3 \mu$ s prior to the train arrival are cleared out during the first 6 of the 20 bunch passages, reabsorbed either in the detector or the vacuum chamber wall. The signal also shows that the cloud development proceeds at the higher density level following the clearing, since it does not return to the level of the signal for a 10-bunch train. The trapping results in a sustained higher cloud density even after the trapped electrons have been removed.

Figure 8 shows the modeled electron cloud density averaged over the test volume of the cylindrical vacuum chamber for the case of a 20-bunch train of positrons with average bunch population $1.3 \times 10^{11}$, with and without an intermediate clearing bunch of the same population.

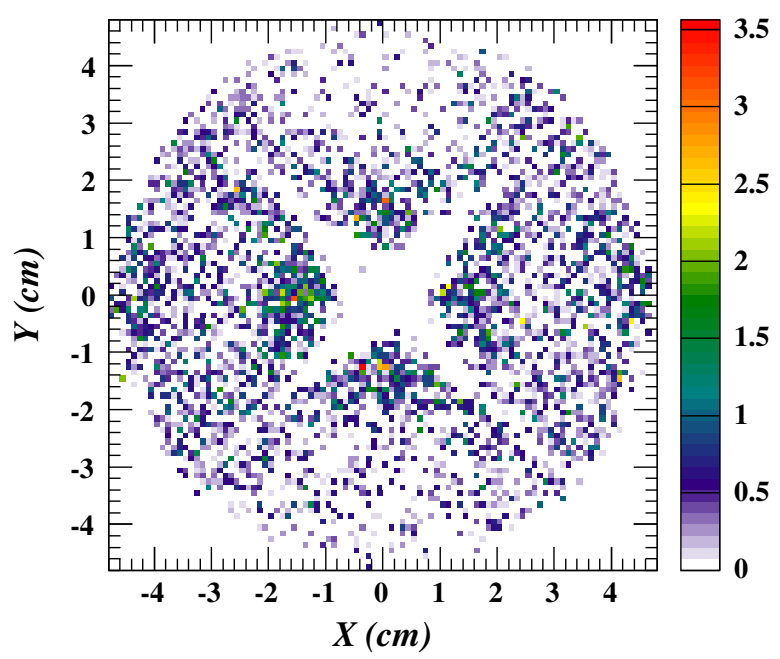

FIG. 9. The modeled transverse distribution of the trapped cloud shown at the end of the first beam revolution. The color scale ranges up to a maximum of $3.5 \times 10^{6}$ electrons/bin. 
The peak density in the absence of the clearing bunch reaches $1.1 \times 10^{12} \mathrm{~m}^{-3}$ after three turns, about $7 \%$ of which is trapped until the train returns. The clearing bunch reduces the trapped cloud density by about a factor of four.

The modeled transverse distribution of the cloud trapped in the quadrupole magnet at a time immediately preceding the return of the train is shown in Fig. 9. The trapped electrons are concentrated in four quadrants near the beam outside of a central depletion zone of $2 \mathrm{~cm}$ diameter, consistent with the trapping probability distribution shown in Fig. 7 and the nonadiabaticity in the central and diagonal regions. The median energy of the trapped electrons is about $50 \mathrm{eV}$.

\section{SUMMARY}

Our measurements with a time-resolving electron detector located in a quadrupole magnetic field have provided comparisons of signals from 10- and 20-bunch trains of positrons which show clear evidence for electron trapping during the entire $2.3 \mu$ s time interval prior to the return of the bunch train. Modeling tuned to the recorded signals indicates that approximately $7 \%$ of the cloud generated by a $5.3 \mathrm{GeV}$ train of 20 bunches, each carrying $1.3 \times 10^{11}$ positrons, remains trapped. The measurements show a nonmonotonic dependence on bunch spacing. The clearing effect of an intermediate bunch has been measured and successfully modeled, showing the trapped cloud can be reduced by a factor of four by such a clearing bunch. This characteristic of a quadrupole magnetic field to concentrate electrons near the beam raises concerns for storage rings with positively charged beams, since those electrons can be attracted into the beam. Such measurements quantifying electron trapping in quadrupole magnets provide information useful for the development of simulation codes which serve to predict electron cloud phenomena in future accelerators and to aid in the design of mitigation techniques.

\section{ACKNOWLEDGMENTS}

We wish to thank Joe Calvey, Gerry Dugan, Miguel Furman and David Rubin for useful discussions. This work is supported by the U.S. National Science Foundation Contracts No. PHY-0734867, No. PHY-1002467, and No. PHY-1068662, by the U.S. Department of Energy Contract No. DE-FC02-08ER41538 and by the Japan/US Cooperation Program.

[1] F. Zimmermann, Electron-cloud effects in past \& future machines_-walk through 50 years of electron-cloud studies, in Proceedings of ECLOUD 2012: Joint INFNCERN-EuCARD-AccNet Workshop on Electron-Cloud Effects, La Biodola, Elba, Italy, CERN-2013-002, edited by R. Cimino, G. Rumolo, and F. Zimmermann (CERN, Geneva, Switzerland, 2013), p. 9.

[2] K. Ohmi, Beam-Photoelectron Interactions in Positron Storage Rings, Phys. Rev. Lett. 75, 1526 (1995).

[3] M. A. Furman and G. R. Lambertson, The electron-cloud instability in PEP-II: An update, in Proceedings of the Particle Accelerator Conference, Vancouver, BC, Canada, 1997 (IEEE, New York, 1997), p. 1617.

[4] R. J. Macek, A. A. Browman, J. E. Ledford, M. J. Borden, J. F. O'Hara, R. C. McCrady, L. J. Rybarcyk, T. Spickermann, T. J. Zaugg, and M. T. F. Pivi, Electron cloud generation and trapping in a quadrupole magnet at the Los Alamos proton storage ring, Phys. Rev. ST Accel. Beams 11, 010101 (2008).

[5] G. Iadarola and G. Rumolo, Electron cloud in the CERN accelerators (PS, SPS, LHC), in Proceedings of ECLOUD 2012: Joint INFN-CERN-EuCARD-AccNet Workshop on Electron-Cloud Effects, La Biodola, Elba, Italy, CERN-2013-002, edited by R. Cimino, G. Rumolo, and F. Zimmermann (CERN, Geneva, Switzerland, 2013) pp. 19-26.

[6] K. Brodzinski and L. Tavian, First measurements of beaminduced heating on the LHC cryogenic system, Report No. CERN-ATS-2013-009 (CERN, Geneva, Switzerland, 2013); in International Cryogenic Engineering Conferencel International Cryogenic Materials Conference, Fukuoka, Japan, May 2012 (Cryogenics and Superconductivity Society of Japan, Tokya, 2012).

[7] Y. Suetsugu, K.-I. Kanazawa, K. Shibata, T. Ishibashi, H. Hisamatsu, M. Shirai, and S. Terui, Design and construction of the SuperKEKB vacuum system, J. Vac. Sci. Technol. A 30, 031602 (2012).

[8] J. A. Crittenden, J. Conway, G. F. Dugan, M. A. Palmer, D. L. Rubin, J. Shanks, K. G. Sonnad, L. Boon, K. Harkay, T. Ishibashi, M. A. Furman, S. Guiducci, M. T. F. Pivi, and L. Wang, Investigation into electron cloud effects in the International Linear Collider positron damping ring, Phys. Rev. ST Accel. Beams 17, 031002 (2014).

[9] L. Rossi, LHC upgrade plans: Options and strategy, in Proceedings of the 2nd International Particle Accelerator Conference, San Sebastián, Spain (EPS-AG, Spain, 2011), p. 908 .

[10] C. Yin Vallgren, P. Chiggiato, P. C. Pinto, H. Neupert, G. Rumolo, E. Shaposhnikova, M. Taborelli, and S. Kato, Performance of carbon coating for mitigation of electron cloud in the SPS, in Proceedings of the 2nd International Particle Accelerator Conference, San Sebastián, Spain (EPS-AG, Spain, 2011), p. 1590.

[11] M. Kireeff Covo, A. W. Molvik, A. Friedman, J.-L. Vay, P. A. Seidl, G. Logan, D. Baca, and J. L. Vujic, Absolute Measurement of Electron-Cloud Density in a Positively Charged Particle Beam, Phys. Rev. Lett. 97, 054801 (2006).

[12] R. Cimino, I. R. Collins, M. A. Furman, M. Pivi, F. Ruggiero, G. Rumolo, and F. Zimmermann, Can Low-Energy Electrons Affect High-Energy Physics Accelerators?, Phys. Rev. Lett. 93, 014801 (2004).

[13] G. Iadarola, Electron cloud studies for CERN particle accelerators and simulation code development, Ph.D. thesis, University of Naples, Naples, Italy, 2014. 
[14] L. F. Wang, H. Fukuma, S. Kurokawa, and K. Oide, Photoelectron trapping in quadrupole and sextupole magnetic fields, Phys. Rev. E 66, 036502 (2002).

[15] L. Wang and M. Pivi, Trapping of electron cloud in ILC/ CESRTA quadrupole and sextupole magnets, in Proceedings of ECLOUD 2010: 49th ICFA Advanced Beam Dynamics Workshop on Electron Cloud Physics, Ithaca, $N Y$, edited by K. Smolenski (Cornell University, Ithaca, NY, 2013), p. 167.

[16] G. F. Dugan, M. A. Palmer, and D. L. Rubin, ILC damping rings $\mathrm{R} \& \mathrm{D}$ at CESRTA, in ICFA Beam Dynamics Newsletter, No. 50, edited by J. Urakawa (International Committee on Future Accelerators, 2009), p. 11.

[17] J. R. Calvey, W. Hartung, Y. Li, J. A. Livezey, J. Makita, M. A. Palmer, and D. Rubin, Comparison of electron cloud mitigating coatings using retarding field analyzers, Nucl. Instrum. Methods Phys. Res., Sect. A 760, 86 (2014).

[18] J. R. Calvey, G. Dugan, W. Hartung, J. A. Livezey, J. Makita, and M. A. Palmer, Measurement and modeling of electron cloud in a field free environment using retarding field analyzers, Phys. Rev. ST Accel. Beams 17, 061001 (2014).

[19] J. A. Crittenden, Y. Li, X. Liu, M. A. Palmer, and J. P. Sikora, Modeling for time-resolved retarding field analyzer measurements of electron cloud buildup at CESRTA, in Proceedings of the 4th International Particle Accelerator Conference, IPAC-2013, Shanghai, China, 2013, edited by Z. Dai, C. Petit-Jean-Genaz, V. R. W. Schaa, and C. Zhang (JACoW, Shanghai, China, 2013), p. 846.

[20] J. A. Crittenden, M. G. Billing, Y. Li, M. A. Palmer, and J. P. Sikora, Shielded button electrodes for time-resolved measurements of electron cloud buildup, Nucl. Instrum. Methods Phys. Res., Sect. A 749, 42 (2014).
[21] J. A. Crittenden and J.P. Sikora, Electron cloud buildup characterization using shielded-pickup measurements and custom modeling code at CESRTA, in Proceedings of ECLOUD 2012: Joint INFN-CERN-EuCARD-AccNet Workshop on Electron-Cloud Effects, La Biodola, Elba, Italy, edited by R. Cimino, G. Rumolo, and F. Zimmermann (CERN, Geneva, Switzerland, 2013), p. 241.

[22] M. Sands, Energy loss from small holes in the vacuum chamber, SLAC, Report No. PEP-253, 1977.

[23] R. J. Goldston and P. H. Rutherford, Introduction to Plasma Physics (Institute of Physics, Bristol, 1995).

[24] E. E. Cowan, K. G. Sonnad, and S. Veitzer, Trajectories of low energy electrons in particle accelerator magnetic structures, in Proceedings of the 25th Particle Accelerator Conference, PAC-2013, Pasadena, CA, 2013, edited by T. Satogata, C. Petit-Jean-Genaz, and V. Schaa (IEEE, New York, 2013), p. 475.

[25] J.S. Berg, Energy gain in an electron cloud during the passage of a bunch, CERN Project Note 97, 1997.

[26] G. Rumolo and F. Zimmermann, Electron cloud simulations: beam instabilities and wakefields, Phys. Rev. ST Accel. Beams 5, 121002 (2002).

[27] M. A. Furman and M. T. F. Pivi, Probabilistic model for the simulation of secondary electron emission, Phys. Rev. ST Accel. Beams 5, 124404 (2002).

[28] J. A. Crittenden, M. G. Billing, W. H. Hartung, C. S. Shill, J. P. Sikora, and K. G. Sonnad, Numerical modeling for CESRTA measurements of electron cloud buildup in a quadrupole magnet, in IPAC2014: Proceedings of the 5th International Particle Accelerator Conference, Dresden, Germany, edited by C. Petit-Jean-Genaz, G. Arduini, P. Michel, and V. R. W. Schaa (JACoW, Geneva, Switzerland, 2014), p. 1632. 